\title{
Evaluation of the effect of salmon nasal proteoglycan on biomarkers for cartilage metabolism in individuals with knee joint discomfort: A randomized double-blind placebo-controlled clinical study
}

\author{
AKIHITO TOMONAGA ${ }^{1}$, TATSUJI TAKAHASHI ${ }^{2}$, YUKA TSUDA TANAKA ${ }^{2}$, \\ MAKOTO TSUBOI $^{2}$, KUMIE ITO ${ }^{3}$ and ISAO NAGAOKA ${ }^{4}$ \\ ${ }^{1}$ Tana Orthopedic Surgery, Yokohama, Kanagawa 227-0064; ${ }^{2}$ Research and Development Department, \\ Ichimaru Pharcos Co., Ltd., Gifu 501-0475; ${ }^{3}$ Nihonbashi Sakura Clinic, Tokyo 103-0025; ${ }^{4}$ Department of \\ Host Defense and Biochemical Research, Graduate School of Medicine, Juntendo University, Tokyo 113-8421, Japan
}

Received April 26, 2016; Accepted February 24, 2017

DOI: $10.3892 /$ etm.2017.4454

\begin{abstract}
A randomized double-blind placebo-controlled clinical trial was conducted to evaluate the chondroprotective action of salmon nasal cartilage proteoglycan on joint health. The effect of oral administration of proteoglycan (10 mg/day) on cartilage metabolism was evaluated in individuals with knee joint discomfort but without diagnosis of knee osteoarthritis. The average age of patients was $52.6 \pm 1.1$ years old. The effect of proteoglycan was evaluated by analyzing markers for type II collagen degradation $(\mathrm{C} 1,2 \mathrm{C})$ and synthesis (PIICP), and the ratio of type II collagen degradation to synthesis. The results indicated that the change in $\mathrm{C} 1,2 \mathrm{C}$ levels significantly differed in the proteoglycan group compared with the placebo group following 16 weeks intervention among subjects with high levels of knee pain and physical dysfunction (total score of Japan Knee Osteoarthritis Measure $\geq 41$ ) and subjects with constant knee pain (both $\mathrm{P}<0.05$ ). There was a greater increase in PIICP levels in the proteoglycan group than the placebo group following intervention, although this difference was not significant in both sets of patients. Thus, the $\mathrm{C} 1,2 \mathrm{C} / \mathrm{PIICP}$ ratios decreased in the proteoglycan group, whereas they slightly increased in the placebo group following the intervention. Furthermore, no test supplement-related adverse events were observed during the intervention. Therefore, oral administration of salmon nasal cartilage proteoglycan at a dose of $10 \mathrm{mg} /$ day may exert a chondroprotective action in subjects with knee joint discomfort. This effect was achieved
\end{abstract}

Correspondence to: Professor Isao Nagaoka, Department of Host Defense and Biochemical Research, Graduate School of Medicine, Juntendo University, 2-1-1 Hongo, Tokyo 113-8421, Japan

E-mail: nagaokai@juntendo.ac.jp

Key words: proteoglycan, biomarker, cartilage metabolism, joint health, salmon by improving cartilage metabolism (reducing type II collagen degradation and enhancing type II collagen synthesis), without causing apparent adverse effects.

\section{Introduction}

Osteoarthritis develops due to the progressive destruction of articular cartilage. It is the most common joint disease and the leading cause of physical dysfunction and pain in elderly people (1-3). In osteoarthritis, knee joints are particularly impaired, as they are weight-bearing joints. Previous results in experimental osteoarthritis models have indicated that changes in the chemical and metabolic properties of cartilage matrix are detectable at the early stages of arthritis (4). Thus, various molecular markers have been developed to measure cartilage metabolism in joint disorders (5-9). Such biomarkers are used to evaluate the actions of disease-modifying drugs and chondroprotective supplements on the cartilage as they specifically reflect alterations in cartilage metabolism (10).

Type II collagen is a major constituent of cartilage and represents $90-95 \%$ of the cartilage collagens (7). Thus, components of type II collagen have been utilized as biomarkers for cartilage metabolism. A C-terminal crosslinking peptide (CTX-II) is cleaved during degradation of type II collagen (11), whereas a neoepitope, either $\mathrm{C} 2 \mathrm{C}$ or $\mathrm{C} 1,2 \mathrm{C} / \mathrm{Col} 23 / 4 \mathrm{C}_{\text {short }}$, is generated by intrahelical cleavage at the $\mathrm{C}$ terminus of the $3 / 4$ piece of degraded type II collagen $(12,13)$. Thus, CTX-II and $\mathrm{C} 2 \mathrm{C}$ or $\mathrm{C} 1,2 \mathrm{C}$ are both used as markers for type II collagen degradation. By contrast, a C-terminal type II procollagen propeptide (PIICP), which is cleaved during the processing of newly synthesized type II procollagen, can be used as a marker for type II collagen synthesis (14). In addition, along with type II collagen, aggrecan is one of the most abundant proteins of the cartilage matrix, and aggrecan synthesis can be measured using antibodies against epitope CS846, which is located on the chondroitin sulfate chains (15). Notably, in the cartilage of patients with osteoarthritis aggrecan levels are significantly increased (16). Furthermore, cartilage 
oligometric matrix protein (COMP), a member of the thrombospondin family of glycoproteins, is predominantly found in the extracellular matrix of cartilage, tendons and ligaments, and it has been demonstrated that serum levels of COMP are increased in individuals with osteoarthritis (17). Thus, CS846 and COMP may also be utilized as markers of cartilage metabolism $(18,19)$.

Various nutritional supplements, including glucosamine, chondroitin and collagen, are used to promote joint health. They are also specifically used to treat or prevent cartilage disorders, such as including osteoarthritis $(20,21)$. Glucosamine suppresses the degradation and stimulates the synthesis of glycosaminoglycans, such as proteoglycans $(22,23)$. Glucosamine also suppresses the expression of collagen-degrading enzymes, such as matrix metalloproteinases (MMPs) and stimulates type II collagen synthesis in chondrocytes $(24,25)$. Thus, studies have suggested that glucosamine may exert chondroprotective action in cartilage disorders by maintaining proteoglycan and type II collagen levels in the articular cartilage (26-28).

A previous open-label (unblended) study by the current authors investigating individuals with knee osteoarthritis indicated that administration of proteoglycan extracted from salmon nasal cartilage alleviates the symptoms of knee osteoarthritis, possibly by reducing the degradation of type II collagen, as evidenced by a reduction in the $\mathrm{C} 2 \mathrm{C} / \mathrm{PIICP}$ ratio (29). Notably, salmon nasal cartilage proteoglycan has the potential to induce the proliferation of chondrocytes and the production of proteoglycans by chondrocytes in vitro $(29,30)$ and also exhibits anti-inflammatory action in vivo in arthritic mice (31). Thus, it was proposed that the administration of salmon nasal cartilage proteoglycan may improve the symptoms of knee osteoarthritis, due to its chondroprotective and anti-inflammatory actions.

In the present study, a randomized double-blind placebo-controlled clinical trial was conducted to evaluate the chondroprotective action of salmon nasal proteoglycan on cartilage metabolism. The effect of oral administration of proteoglycan $(10 \mathrm{mg} /$ day $)$ on cartilage metabolism was investigated in subjects with knee joint discomfort by analyzing serum levels of markers for type II collagen $(\mathrm{C} 1,2 \mathrm{C}$ and PIICP), aggrecan (CS846) and COMP. The results indicated that administration of salmon nasal cartilage proteoglycan may suppress the degradation of type II collagen, as evidenced by the significant reduction of $\mathrm{C} 1,2 \mathrm{C}$ level, in the cartilage of subjects with severe or constant joint discomfort.

\section{Materials and methods}

Subjects. Inclusion criteria were as follows: i) Japanese male or female aged 35-75 years; ii) radiographic severity of knee joints grade 0 or 1, based on Kellgren and Lawrence grades (32) (grade 0, no radiographic features of osteoarthritis; grade 1, doubtful joint space narrowing and possible osteophytic lipping) without the diagnosis of osteoarthritis by orthopedists; iii) one knee joint with visual analog scale (VAS) of $\geq 20 \mathrm{~mm}$ (33), based on at least one of four VAS subscales, including subscale I (degree of knee pain evaluated by VAS) of the Japan Knee Osteoarthritis Measure (JKOM) (33) and three VAS subscales (pain at rest, pain at walking and pain at going up and down stairs). Each VAS subscale was scored from 0 to $100 \mathrm{~mm}$, where $0 \mathrm{~mm}$ indicates 'no pain at all' and $100 \mathrm{~mm}$ indicates 'the most severe pain ever experienced'.

Exclusion criteria were as follows: i) Total VAS subscale of ankle, elbow, shoulder and hip joints being greater than the subscale of knee joint (the subscale I of JKOM); ii) a score of $\geq 30$ points on subscale I (pain on walking) of the Japanese Orthopedic Association criteria (34), which indicates that patients can walk $\geq 1 \mathrm{~km}$ without apparent pain; iii) a diagnosis of gout/hyperuricemia or rheumatoid arthritis; iv) surgical treatment of joint(s) performed or required; v) clinical history of bone or cartilage disorders including fracture and distortion within one year prior to enrollment; vi) routine use of dietary supplements containing proteoglycans, hyaluronic acid, $\mathrm{N}$-acetyl glucosamine, glucosamine, chondroitin sulfate, collagen peptides or any other constituents of the test supplement within three months prior to enrollment; vii) hypersensitivity or allergy to the test component; viii) diagnosis or current medication of disorders including malignancies, hypertension (atherosclerosis), cardiac, renal, thyroid, lung and hepatic disorders, or cerebral infarction; ix) routine use of external medicine including poultices and taking prescribed medicine ( $>3$ days/week); $x$ ) intra-articular injections of either corticosteroids or hyaluronic acid within one year prior to enrollment; xi) excessive exercise, which places loads on the joints; xii) daily drinking of $>60 \mathrm{~g}$ alcohol/day; xiii) pregnant women, nursing mothers or women intending to have children in the future; xiv) participation in any other clinical studies within one month prior to enrollment; and xv) the presence of any clinical conditions judged by the medical investigator to preclude the participation of subjects in the study.

Following assessment of 180 subjects for eligibility, 115 subjects were excluded based on the exclusion criteria and 5 subjects declined to participate of their own volition. Finally, 60 Japanese adults (26 males and 34 females; aged 36-71 years; mean age, 52.4 \pm 1.1 years) with knee discomfort but without a diagnosis of knee osteoarthritis (Kellgren and Lawrence grades 0-1) (32) were enrolled as eligible subjects. The research co-coordinators created an allocation table and randomly assigned the eligible subjects to receive a salmon nasal proteoglycan-containing capsule $(n=30$, proteoglycan group) or a placebo capsule ( $\mathrm{n}=30$, placebo group; Fig. 1). The allocation table was sealed, and all research staff and participants were blinded to the allocation during the test period. Following completion of the study, the allocation table was made available for analysis of the data. During the intervention, two subjects in the placebo group discontinued the study of their own volition (one for a long-term business trip, and the other for the treatment of right knee joint pain). A further three subjects (two subjects in the placebo group and one subject in the proteoglycan group) were excluded by the medical investigator, due to the onset of renal stone and joint disease, taking prescribed medicine including inhaled steroids and the use of external medicine (non-steroidal anti-inflammatory drug-containing poultice) for lower back pain during the intervention, which may have affected the efficacy of test supplements. Thus, 55 subjects (mean age, $52.6 \pm 1.1$ years; $\mathrm{n}=26$ in the placebo group, $\mathrm{n}=29$ in the proteoglycan group) were judged to be eligible for assessment of the efficacy of the test supplement (Fig. 1 and Table I). 


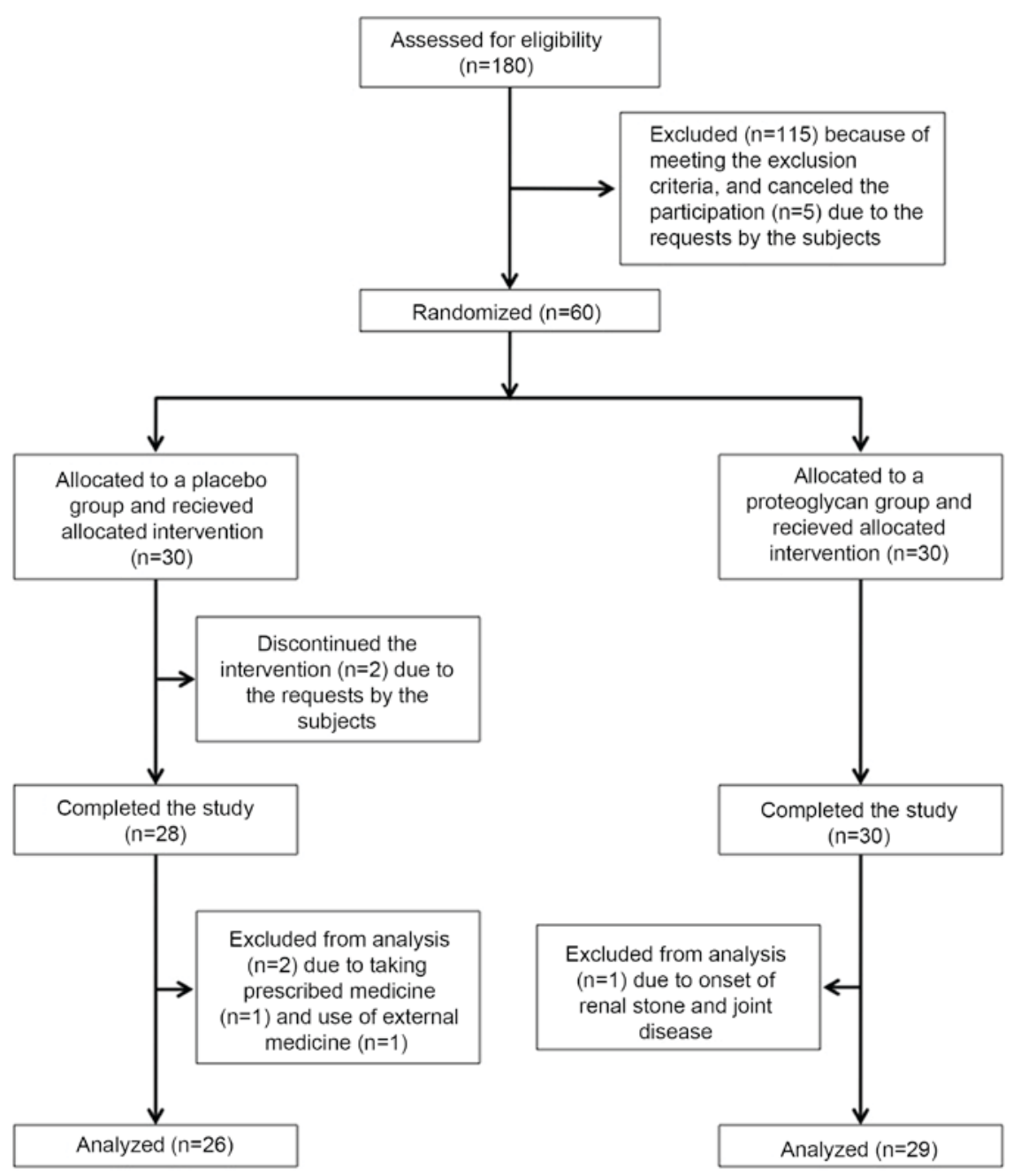

Figure 1. Flow diagram of the subjects who participated in the study.

Study design. A prospective randomized double-blind placebo-controlled, parallel-group comparative study was designed to compare the actions of salmon nasal cartilage proteoglycan and a placebo on cartilage metabolism. Markers for type II collagen synthesis (PIICP) and degradation $(\mathrm{C} 1,2 \mathrm{C})$, aggrecan (CS846) and COMP in individuals with knee joint discomfort were measured. In addition, the safety of the test was evaluated throughout. Subjects were enrolled and screened from February 2015 to April 2015, and the test supplements were administered from May 2015 to August 2015 in the Nihonbashi Sakura Clinic (Tokyo, Japan). The study protocol was registered at the UMIN Clinical Trials Registry (trial no. UMIN000016470), and involved the Nihonbashi Sakura Clinic. The study protocol was approved by the Aisei Hospital Ueno Clinic Research Ethics Committee (Tokyo, Japan) and was conducted in accordance with the principles of the amended Declaration of Helsinki and Ethical Guidelines for Epidemiological Research (established by the Japanese Government in 2008). Written informed consent was obtained from all participants prior to their enrollment in the study. The study consisted of a 4-week run-in (screening) period and a 16-week intervention period. Subjects were screened at a baseline visit by physical examination, knee radiograph according to a standardized method, a symptom questionnaire and routine laboratory tests. In addition, medical examinations were performed at weeks 4, 8, 12 and 16 during the intervention, and laboratory tests were performed at weeks 12 and 16 during the intervention for the enrolled subjects.

Intervention and subject assignment. The test supplement was manufactured in the form of a hard capsule $(220 \mathrm{mg}$ in a capsule) by Ichimaru Pharcos, Co., Ltd. (Gifu, Japan) and consisted of $12.5 \mathrm{mg}$ salmon nasal cartilage extract (containing $10 \mathrm{mg}$ proteoglycan) and $207.5 \mathrm{mg}$ dextrin (as a vehicle). The placebo capsule contained only dextrin powder (Ichimaru Pharcos, Co., Ltd.). Salmon proteoglycan was extracted from salmon (Oncorhynchus keta) nasal cartilage, as previously reported (35). Briefly, frozen $\left(-20^{\circ} \mathrm{C}\right)$ salmon nasal cartilage was dissolved in a solution of $4-5 \%$ acetic acid and salmon proteoglycan was extracted. High-performance liquid chromatography analysis was performed and detected a salmon proteoglycan peak as 450,000 molecular size, as previously reported $(36,37)$.

Subjects were randomly assigned to receive a $10-\mathrm{mg}$ salmon nasal cartilage proteoglycan-containing capsule (proteoglycan group) or a placebo capsule containing only vehicle (placebo group). All subjects were instructed to take the test supplement or placebo with water once a day between 
Table I. Baseline characteristics of subjects in the placebo and PG groups.

\begin{tabular}{|c|c|c|c|}
\hline Variable & Placebo $(n=26)$ & PG (n=29) & P-value \\
\hline Age (years) & $53.9 \pm 1.9$ & $51.3 \pm 1.2$ & 0.3 \\
\hline Male/female (n) & $10 / 16$ & $13 / 16$ & 0.8 \\
\hline Height $(\mathrm{cm})$ & $163.1 \pm 1.8$ & $164.1 \pm 1.3$ & 0.6 \\
\hline Weight (kg) & $57.0 \pm 2.1$ & $58.2 \pm 2.0$ & 0.7 \\
\hline Body mass index $\left(\mathrm{kg} / \mathrm{m}^{2}\right)$ & $21.3 \pm 0.5$ & $21.5 \pm 0.5$ & 0.7 \\
\hline Systolic blood pressure (mmHg) & $112.5 \pm 2.1$ & $111.6 \pm 2.2$ & 0.8 \\
\hline Diastolic blood pressure (mmHg) & $72.1 \pm 1.6$ & $71.0 \pm 1.4$ & 0.6 \\
\hline Pulse rate (beats/min) & $71.5 \pm 1.6$ & $69.7 \pm 1.9$ & 0.5 \\
\hline \multicolumn{4}{|l|}{ Kellgren and Lawrence grade, 0:1 } \\
\hline Right knee (n) & $16: 10$ & $14: 15$ & 0.4 \\
\hline Left knee (n) & $14: 12$ & $14: 15$ & 0.8 \\
\hline JKOM (total score) & $46.3 \pm 1.6$ & $46.5 \pm 1.7$ & 0.8 \\
\hline \multicolumn{4}{|l|}{ VAS (mm) } \\
\hline Pain at rest & $16.6 \pm 5.4$ & $23.8 \pm 4.6$ & 0.3 \\
\hline Pain at walking & $49.2 \pm 4.0$ & $53.2 \pm 3.2$ & 0.4 \\
\hline Pain at going up and down stairs & $61.3 \pm 3.3$ & $62.4 \pm 3.2$ & 0.8 \\
\hline $\mathrm{C} 1,2 \mathrm{C}\left(\mu \mathrm{g} / \mathrm{ml} \mathrm{x} 10^{-1}\right)$ & $7.7 \pm 0.4$ & $7.9 \pm 0.3$ & 0.7 \\
\hline PIICP (ng/ml) & $47.2 \pm 2.0$ & $47.7 \pm 2.2$ & 0.9 \\
\hline $\mathrm{C} 1,2 \mathrm{C} / \mathrm{PIICP}$ ratio $\left(\mathrm{x} 10^{-2}\right)$ & $1.7 \pm 0.1$ & $1.8 \pm 0.1$ & 0.8 \\
\hline CS846 (ng/ml) & $154.8 \pm 13.8$ & $160.7 \pm 16.0$ & 0.8 \\
\hline COMP (ng/ml) & $154.1 \pm 10.2$ & $174.9 \pm 32.9$ & 0.6 \\
\hline
\end{tabular}

PG, proteoglycan; JKOM, Japan Knee Osteoarthritis Measure; VAS, visual analog scale; PIICP, C-terminal type II procollagen propeptide; COMP, cartilage oligometric matrix protein; $\mathrm{C} 1,2 \mathrm{C}$, marker of type II collagen degradation.

breakfast and lunch for 16 weeks. The daily dose of salmon nasal cartilage proteoglycan (10 $\mathrm{mg} /$ day) was determined based on the results of a previous study (29). Adherence to the intervention was evaluated on the basis of a consumption record in the study diary and $<80 \%$ adherence was considered a protocol violation.

Serum and second void of morning urine were collected from the subjects in a fasting state at baseline, and at weeks 12 and 16 during the intervention. Blood samples were collected by venipuncture, and incubated for 20-30 $\mathrm{min}$ at $25^{\circ} \mathrm{C}$, followed by centrifugation at $1,000 \times \mathrm{g}$ for $10 \mathrm{~min}$ at $4^{\circ} \mathrm{C}$ to isolate sera. Serum and urine samples were immediately used for routine laboratory tests; sera were also aliquoted and stored at $-80^{\circ} \mathrm{C}$ until the assays for $\mathrm{C} 1,2 \mathrm{C}$, PIICP, CS846 and COMP were conducted.

Evaluation of cartilage metabolism. To evaluate the effect of the test supplement on cartilage metabolism, the levels of type II collagen degradation $(\mathrm{C} 1,2 \mathrm{C})$ and synthesis (PIICP) markers, CS846 and COMP were evaluated in serum samples. Serum C1,2C and PIICP were measured using a Collagen Type I and II Cleavage ELISA kit (60-1002-001; IBEX Technologies Inc., Montreal, QC, Canada) and ELISA kit for Procollagen II C-Terminal Propeptide (SEA964Hu; Wuhan USCN Business Co., Ltd., Wuhan, China), respectively. The $\mathrm{C} 1,2 \mathrm{C} / \mathrm{PIICP}$ ratio was then calculated. Serum CS846 and COMP were measured using an Aggrecan Chondroitin Sulfate 846 Epitope ELISA kit (60-1004; IBEX Technologies, Inc.) and Human COMP Quantikine ELISA kit (DCMP0; R\&D Systems, Inc., Minneapolis, MN, USA), respectively.

Evaluation of knee pain. The efficacy of the test supplement was also evaluated based on the changes in subscale scores of JKOM (33). This was measured at baseline, and at weeks 4, 8, 12 and 16 during the intervention. JKOM is a self-answered evaluation questionnaire that includes five subcategories: i) Degree of knee pain as determined by VAS; ii) Pain and stiffness in knees (8 questions); iii) Condition in daily life (10 questions); iv) General activities (5 questions); and v) Health conditions (2 questions). The responses to each question (ii-v subcategories) are scored from 1 to 5 points, with 1 point indicating the best functional status and 5 points indicating the worst functional status. The JKOM score is higher in subjects with increased pain and physical dysfunction, and is reliable and valid for studying the clinical outcomes of knee osteoarthritis (38). The outcome of JKOM is closely correlated with that of other arthritis-related scales, including the Western Ontario and McMaster Universities Arthritis Index (38). 
A

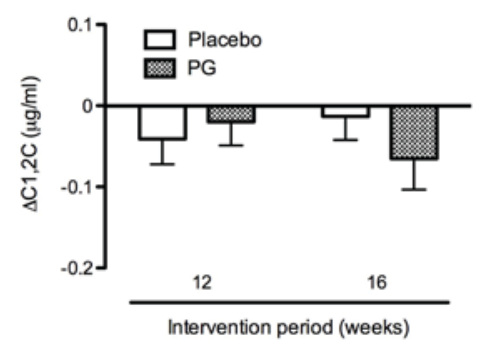

C

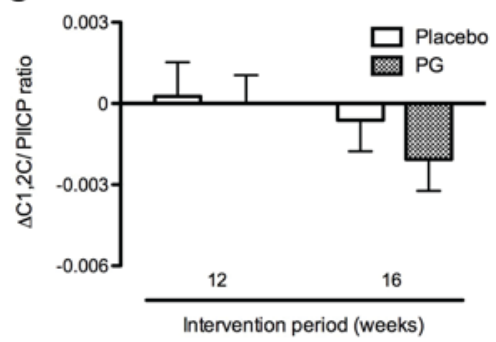

B

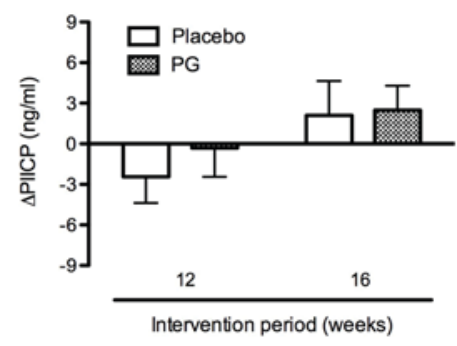

D

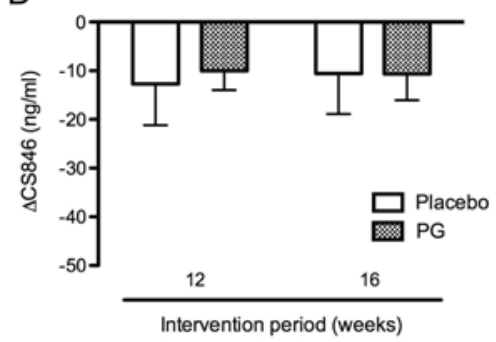

E

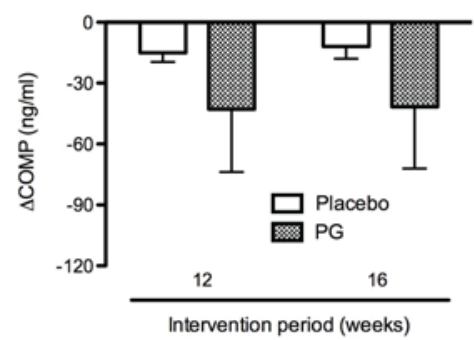

Figure 2. Cartilage metabolism in the placebo and proteoglycan groups during the intervention. Markers of cartilage metabolism were measured using sera collected from the subjects in the placebo $(n=26)$ and proteoglycan $(n=29)$ groups at baseline and at weeks 12 and 16 of the intervention. Marker levels were expressed as changes from the baseline. (A) $\Delta \mathrm{C} 1,2 \mathrm{C}$, (B) $\Delta \mathrm{PIICP},(\mathrm{C}) \Delta \mathrm{C} 1,2 \mathrm{C} / \mathrm{PIICP}$ ratio, (D) $\Delta \mathrm{CS} 486$ and (E) $\Delta \mathrm{COMP}$. PIICP, C-terminal type II procollagen propeptide; COMP, cartilage oligometric matrix protein; $\mathrm{C} 1,2 \mathrm{C}$, marker of type II collagen degradation.

Safety evaluation. Safety and tolerability were assessed throughout the study. The incidence and severity of intervention-related adverse events (side effects) were recorded, as well as abnormal changes in physical parameters, such as blood pressure and pulse rate. The results of laboratory tests, including hematology, biochemical profile and urinalysis, were also recorded. Any changes in physical conditions and the use of pharmaceutical products were recorded in a diary by the enrolled subjects.

Statistical analysis. Data are expressed as the mean \pm standard error of the mean, unless otherwise noted. Regarding the baseline characteristics of subjects, the distributions of males and females, and Kellgren and Lawrence grades were analyzed using Pearson's $\chi^{2}$ test, and other parameters were analyzed using the Student's t-test. Subcategory I of JKOM and VAS subscales were compared using the Student's t-test. Subcategories II-V of JKOM were compared between different time points using the Wilcoxon rank sum test, and between different groups using the Mann Whitney-U test. Safety data, C1,2C, PIICP, CS846 and COMP levels, and $\mathrm{C} 1,2 \mathrm{C} / \mathrm{PIICP}$ ratios were compared using the Student's t-test. $\mathrm{P}<0.05$ was considered to indicate a statistically significant difference.

\section{Results}

Characterization of study groups. Table I presents the baseline characteristics of the 55 subjects ( $n=26$ in the placebo group; $\mathrm{n}=29$ in the proteoglycan group) who completed the study and fulfilled the eligibility criteria. The baseline characteristics included demographic characteristics (age, and distribution of male and female subjects), physiological measurement parameters (body height, body weight and body mass index), physiological examinations (systolic blood pressure, diastolic blood pressure and pulse rate), distribution of Kellgren and Lawrence grades, JKOM (total score), VAS subscales (pain at rest, pain at walking, and pain at going up and down stairs), and levels of biomarkers for type II collagen metabolism $(\mathrm{C} 1,2 \mathrm{C}$, PIICP and C1,2C/PIICP ratio), CS846 and COMP. There were no significant differences in these parameters between the placebo and proteoglycan groups at the baseline. Adherence to the allotted supplement was 96-100\% among the 58 subjects who completed the study.

Assessment of cartilage metabolism using markers for type II collagen degradation and synthesis, CS846 and COMP. In addition to the markers for type II collagen degradation (C1,2C) and synthesis (PIICP), aggrecan (CS846) and COMP, 
Table II. Baseline characteristics of the subjects with a total JKOM score $\geq 41$ points in the placebo and PG groups.

\begin{tabular}{|c|c|c|c|}
\hline Variable & Placebo $(n=19)$ & PG (n=22) & P-value \\
\hline Age (years) & $53.4 \pm 2.1$ & $51.3 \pm 1.2$ & 0.4 \\
\hline Male/female (n) & $8 / 11$ & $13 / 9$ & 0.4 \\
\hline Height $(\mathrm{cm})$ & $163.1 \pm 2.1$ & $165.5 \pm 1.5$ & 0.3 \\
\hline Weight (kg) & $56.9 \pm 2.5$ & $60.6 \pm 2.4$ & 0.3 \\
\hline Body mass index $\left(\mathrm{kg} / \mathrm{m}^{2}\right)$ & $21.2 \pm 0.6$ & $22.0 \pm 0.6$ & 0.4 \\
\hline Systolic blood pressure (mmHg) & $114.7 \pm 2.2$ & $111.6 \pm 2.2$ & 0.3 \\
\hline Diastolic blood pressure (mmHg) & $73.4 \pm 1.7$ & $71.0 \pm 1.6$ & 0.3 \\
\hline Pulse rate (beats/min) & $71.8 \pm 2.0$ & $69.3 \pm 2.0$ & 0.4 \\
\hline \multicolumn{4}{|l|}{ Kellgren and Lawrence grade, 0:1 } \\
\hline Right knee (n) & $13: 06$ & $11: 11$ & 0.3 \\
\hline Left knee (n) & 10:09 & $11: 11$ & $>0.9$ \\
\hline JKOM (total score) & $49.4 \pm 1.7$ & $50.1 \pm 1.6$ & 0.7 \\
\hline \multicolumn{4}{|l|}{ VAS (mm) } \\
\hline Pain at rest & $13.6 \pm 5.1$ & $24.7 \pm 5.2$ & 0.1 \\
\hline Pain at walking & $48.5 \pm 4.4$ & $51.5 \pm 4.1$ & 0.6 \\
\hline Pain at going up and down stairs & $63.9 \pm 3.5$ & $61.7 \pm 4.0$ & 0.7 \\
\hline $\mathrm{C} 1,2 \mathrm{C}\left(\mu \mathrm{g} / \mathrm{ml} \times 10^{-1}\right)$ & $7.4 \pm 0.4$ & $8.0 \pm 0.4$ & 0.3 \\
\hline PIICP (ng/ml) & $47.3 \pm 2.3$ & $48.0 \pm 2.6$ & 0.8 \\
\hline $\mathrm{C} 1,2 \mathrm{C} / \mathrm{PIICP}$ ratio $\left(\mathrm{x} 10^{-2}\right)$ & $1.7 \pm 0.1$ & $1.8 \pm 0.2$ & 0.5 \\
\hline CS846 (ng/ml) & $165.4 \pm 18.1$ & $158.4 \pm 18.9$ & 0.8 \\
\hline COMP (ng/ml) & $160.5 \pm 13.5$ & $190.2 \pm 42.7$ & 0.5 \\
\hline
\end{tabular}

PG, proteoglycan; JKOM, Japan Knee Osteoarthritis Measure; VAS, visual analog scale; PIICP, C-terminal type II procollagen propeptide; COMP, cartilage oligometric matrix protein; $\mathrm{C} 1,2 \mathrm{C}$, marker of type II collagen degradation.

the ratio of type II collagen degradation to synthesis may be used to predict the progression of joint damage in patients with knee osteoarthritis $(39,40)$. The effect of salmon cartilage proteoglycan on cartilage metabolism was evaluated by measuring the $\mathrm{C} 1,2 \mathrm{C} / \mathrm{PIICP}$ ratio as well as levels of $\mathrm{C} 1,2 \mathrm{C}$, PIICP, CS846 and COMP using sera collected at baseline, and at weeks 12 and 16 of the intervention. The levels of $\mathrm{C} 1,2 \mathrm{C}$ and PIICP did not significantly change in the placebo and proteoglycan groups after 16 weeks intervention. There were also no significant differences in $\mathrm{C} 1,2 \mathrm{C}$ and PIICP levels between the placebo and proteoglycan groups. However, $\mathrm{C} 1,2 \mathrm{C}$ levels slightly decreased from baseline levels of $0.77 \pm 0.04$ and $0.79 \pm 0.03 \mu \mathrm{g} / \mathrm{ml}$ to $0.76 \pm 0.03$ and $0.73 \pm 0.03 \mu \mathrm{g} / \mathrm{ml}$ after the intervention for 16 weeks in the placebo and proteoglycan groups, respectively. Thus, C1,2C levels decreased slightly more in the proteoglycan group $(-0.06 \pm 0.04 \mu \mathrm{g} / \mathrm{ml})$ compared with the placebo group $(-0.01 \pm 0.03 \mu \mathrm{g} / \mathrm{ml})$ after the intervention for 16 weeks (Fig. 2A). By contrast, PIICP levels were marginally increased from the baseline levels of 47.20 \pm 2.00 and $47.66 \pm 2.15 \mathrm{ng} / \mathrm{ml}$ to $49.31 \pm 2.57$ and $50.14 \pm 2.58 \mathrm{ng} / \mathrm{ml}$ after the intervention for 16 weeks in the placebo and proteoglycan groups, respectively. PIICP levels increased slightly more in the proteoglycan group $(2.49 \pm 1.80 \mathrm{ng} / \mathrm{ml})$ compared with the placebo group $(2.11 \pm 2.52 \mathrm{ng} / \mathrm{ml})$ after 16 weeks intervention, although this difference was not significant
(Fig. 2B). Furthermore, the C1,2C/PIICP ratios decreased from the baseline after 16 weeks intervention in both groups. The $\mathrm{C} 1,2 \mathrm{C} / \mathrm{PIICP}$ ratios decreased slightly more in the proteoglycan group $(-0.002 \pm 0.001)$ compared with the placebo group $(-0.001 \pm 0.001)$ after the intervention for 16 weeks (Fig. 2C), although there was no significant difference between the two groups.

Levels of CS846 were not markedly altered during the intervention period in the placebo and proteoglycan groups (Fig. 2D), while levels of COMP decreased somewhat more in the proteoglycan group than in the placebo group (Fig. 2E). However, there were no significant differences in COMP levels between the placebo and proteoglycan groups at weeks 12 and 16 .

Assessment of cartilage metabolism in subjects with severe knee pain. In order to improve clarity regarding the effect of the test supplement, further assessments focused on subjects with a high level of pain and physical dysfunction, as determined by their JKOM score (33). Subjects with a total JKOM score of $<41$ ( $25 \%$ of the 55 subjects) were excluded. Thus, 41 subjects (mean age, $52.3 \pm 1.2$ years; $n=19$ in placebo group, $\mathrm{n}=22$ in proteoglycan group) with a total score of JKOM $\geq 41$ (increased pain and physical dysfunction) were evaluated. Table II presents the baseline characteristics of these 
A

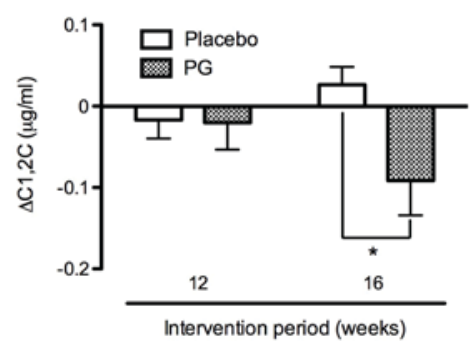

C

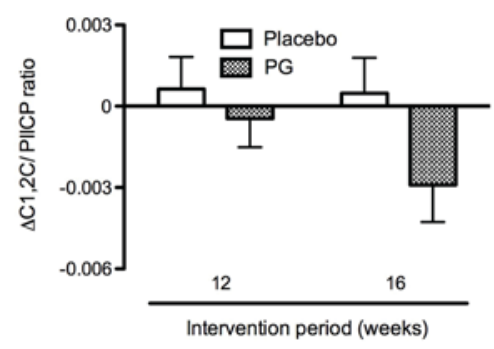

B

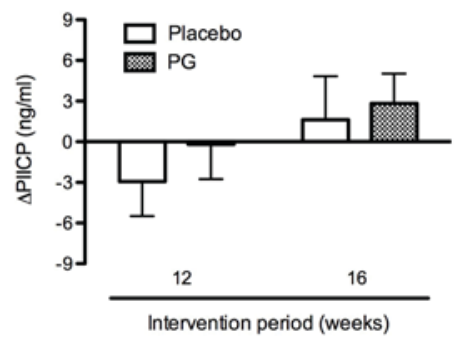

D

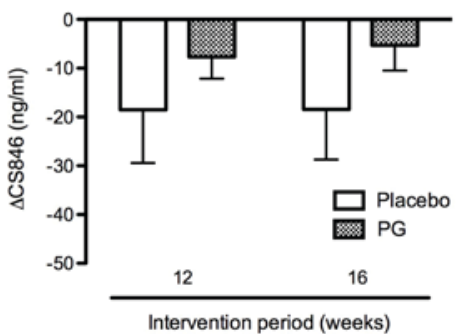

E

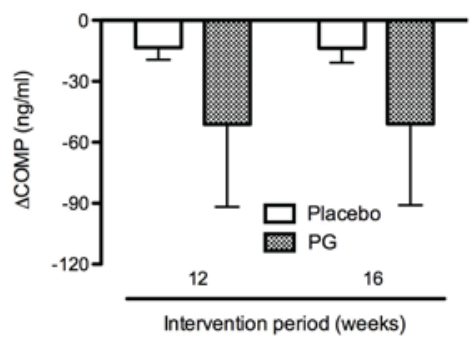

Figure 3. Cartilage metabolism in subjects with total Japan Knee Osteoarthritis Measure score $\geq 41$. Markers of cartilage metabolism were measured using sera collected from the subjects in the placebo $(n=19)$ and proteoglycan $(n=22)$ groups at baseline and at weeks 12 and 16 of the intervention. The marker levels were expressed as changes from the baseline. (A) $\Delta \mathrm{C} 1,2 \mathrm{C}$, (B) $\Delta \mathrm{PIICP},(\mathrm{C}) \Delta \mathrm{C} 1,2 \mathrm{C} / \mathrm{PIICP}$ ratio, (D) $\Delta \mathrm{CS} 486$ and (E) $\Delta \mathrm{COMP}$. ${ }^{*}<0.05$ vs. placebo. PIICP, C-terminal type II procollagen propeptide; COMP, cartilage oligometric matrix protein; $\mathrm{C} 1,2 \mathrm{C}$, marker of type II collagen degradation.

subjects, including demographic characteristics, physiological characteristics, distribution of Kellgren and Lawrence grades, JKOM (total score), VAS subscales and levels of biomarkers. There were no significant differences between these parameters in the placebo and proteoglycan groups.

In the proteoglycan group, $\mathrm{C} 1,2 \mathrm{C}$ levels decreased from the baseline levels of $0.80 \pm 0.04$ to $0.71 \pm 0.03 \mu \mathrm{g} / \mathrm{ml}$ after 16 weeks intervention. In the placebo group, $\mathrm{C} 1,2 \mathrm{C}$ levels increased from the baseline levels of $0.74 \pm 0.04$ to $0.77 \pm 0.04 \mu \mathrm{g} / \mathrm{ml}$ after 16 weeks intervention in the placebo group. Thus, the $\mathrm{C} 1,2 \mathrm{C}$ levels were significantly decreased in the proteoglycan group $(-0.09 \pm 0.04 \mu \mathrm{g} / \mathrm{ml})$ compared with the placebo group $(0.03 \pm 0.02 \mu \mathrm{g} / \mathrm{ml})$ after 16 weeks intervention $(\mathrm{P}<0.05$; Fig. 3A). By contrast, PIICP levels were slightly increased from the baseline levels of $47.34 \pm 2.30$ and $48.03 \pm 2.55 \mathrm{ng} / \mathrm{ml}$ to $48.96 \pm 3.14$ and $50.86 \pm 3.12 \mathrm{ng} / \mathrm{ml}$ in the placebo and proteoglycan groups, respectively following 16 weeks intervention. PIICP levels increased slightly more in the proteoglycan group $(2.83 \pm 2.20 \mathrm{ng} / \mathrm{ml})$ compared with the placebo group $(1.63 \pm 3.21 \mathrm{ng} / \mathrm{ml})$ after 16 weeks intervention (Fig. 3B). Furthermore, C1,2C/PIICP ratios were markedly decreased in the proteoglycan group $(-0.003 \pm 0.001)$ compared with the placebo group $(0.001 \pm 0.001)$ after 16 weeks intervention, though this difference was not significant (Fig. 3C).
Levels of CS846 were markedly decreased during the intervention period in the placebo group relative to the proteoglycan group (Fig. 3D), while levels of COMP were reduced to a greater extent in the proteoglycan group than in the placebo group (Fig. 3E). However, levels of CS846 and COMP did not differ significantly between the placebo and proteoglycan groups at weeks 12 and 16 .

Assessment of cartilage metabolism in subjects with constant knee pain. The effect of the test supplement was also evaluated in subjects with constant knee joint discomfort, based on the subscale of VAS (pain at rest). Thus, 25 subjects without pain at rest (VAS subscale of 0 ) were excluded. A total of 30 subjects (mean age, $50.1 \pm 1.4$ years $n=12$ in the placebo group and $n=18$ in the proteoglycan group) with a subscale of VAS (pain at rest) $\geq 1 \mathrm{~mm}$ were evaluated. Table III presents the baseline characteristics of these subjects. No significant differences in these parameters were found between the placebo and proteoglycan groups.

In the proteoglycan group, $\mathrm{C} 1,2 \mathrm{C}$ levels decreased from the baseline level of $0.80 \pm 0.04$ to $0.70 \pm 0.03 \mu \mathrm{g} / \mathrm{ml}$ after 16 weeks intervention. In the placebo group, $\mathrm{C} 1,2 \mathrm{C}$ levels increased from the baseline levels of $0.79 \pm 0.04$ to $0.83 \pm 0.05 \mu \mathrm{g} / \mathrm{ml}$ after 16 weeks intervention. Thus, $\mathrm{C} 1,2 \mathrm{C}$ levels were significantly 
Table III. Baseline characteristics of subjects with knee pain at rest in the placebo and PG groups.

\begin{tabular}{|c|c|c|c|}
\hline Variable & Placebo $(\mathrm{n}=12)$ & PG (n=18) & P-value \\
\hline Age (years) & $49.6 \pm 2.9$ & $50.5 \pm 1.4$ & 0.9 \\
\hline Male/female (n) & $5 / 7$ & $8 / 10$ & $>0.9$ \\
\hline Height (cm) & $164.9 \pm 3.2$ & $163.4 \pm 1.4$ & 0.9 \\
\hline Weight (kg) & $56.3 \pm 3.2$ & $57.2 \pm 2.2$ & 0.8 \\
\hline Body mass index $\left(\mathrm{kg} / \mathrm{m}^{2}\right)$ & $20.7 \pm 0.6$ & $21.3 \pm 0.7$ & 0.5 \\
\hline Systolic blood pressure (mmHg) & $113.4 \pm 3.6$ & $110.0 \pm 2.5$ & 0.4 \\
\hline Diastolic blood pressure (mmHg) & $72.3 \pm 2.8$ & $70.1 \pm 1.4$ & 0.4 \\
\hline Pulse rate (beats/min) & $70.0 \pm 2.5$ & $69.4 \pm 2.1$ & 0.9 \\
\hline \multicolumn{4}{|l|}{ Kellgren and Lawrence grade, $0: 1$} \\
\hline Right knee (number of knees) & 06:06 & 09:09 & $>0.9$ \\
\hline Left knee (number of knees) & $05: 07$ & 08:10 & $>0.9$ \\
\hline JKOM (total score) & $48.8 \pm 3.1$ & $47.7 \pm 2.4$ & 0.9 \\
\hline \multicolumn{4}{|l|}{ VAS (mm) } \\
\hline Pain at rest & $35.9 \pm 9.0$ & $38.3 \pm 4.9$ & 0.8 \\
\hline Pain at walking & $53.5 \pm 6.8$ & $54.6 \pm 3.9$ & 0.9 \\
\hline Pain at going up and down stairs & $62.1 \pm 4.7$ & $61.2 \pm 3.9$ & 0.9 \\
\hline $\mathrm{C} 1,2 \mathrm{C}\left(\mu \mathrm{g} / \mathrm{ml} \times 10^{-1}\right)$ & $7.9 \pm 0.4$ & $8.0 \pm 0.4$ & $>0.9$ \\
\hline PIICP (ng/ml) & $48.4 \pm 2.8$ & $47.5 \pm 2.6$ & 0.8 \\
\hline $\mathrm{C} 1,2 \mathrm{C} / \mathrm{PIICP}$ ratio $\left(\mathrm{x} 10^{-2}\right)$ & $1.7 \pm 0.2$ & $1.8 \pm 0.1$ & 0.8 \\
\hline CS846 (ng/ml) & $173.5 \pm 26.8$ & $162.8 \pm 24.4$ & 0.8 \\
\hline COMP (ng/ml) & $142.6 \pm 16.2$ & $189.4 \pm 52.5$ & 0.5 \\
\hline
\end{tabular}

PG, proteoglycan; JKOM, Japan Knee Osteoarthritis Measure; VAS, visual analog scale; PIICP, C-terminal type II procollagen propeptide; COMP, cartilage oligometric matrix protein; $\mathrm{C} 1,2 \mathrm{C}$, marker of type II collagen degradation.

decreased in the proteoglycan group $(-0.10 \pm 0.05 \mu \mathrm{g} / \mathrm{ml})$ compared with the placebo group $(0.03 \pm 0.02 \mu \mathrm{g} / \mathrm{ml})$ after 16 weeks intervention ( $\mathrm{P}<0.05$; Fig. $4 \mathrm{~A})$. By contrast, PIICP levels were slightly increased from the baseline levels of $48.37 \pm 2.77$ and $47.46 \pm 2.59 \mathrm{ng} / \mathrm{ml}$ to $49.70 \pm 3.89$ and $52.42 \pm 3.69 \mathrm{ng} / \mathrm{ml}$ in the placebo and proteoglycan groups respectively, after 16 weeks intervention. Thus, PIICP levels from the baseline were slightly increased in the proteoglycan group $(4.96 \pm 2.64 \mathrm{ng} / \mathrm{ml})$ compared with the placebo group $(1.33 \pm 3.90 \mathrm{ng} / \mathrm{ml})$ after 16 weeks intervention (Fig. 4B). Furthermore, the $\mathrm{C} 1,2 \mathrm{C} / \mathrm{PIICP}$ ratio decreased in the proteoglycan group $(-0.003 \pm 0.001)$ whereas it increased in the placebo group $(0.001 \pm 0.002)$ after 16 weeks intervention, although this difference was not significant (Fig. 4C).

By contrast, levels of CS846 were markedly decreased during the intervention period in the placebo group relative to the proteoglycan group (Fig. 4D), while levels of COMP were reduced to a greater extent in the proteoglycan group than in the placebo group (Fig. 4E). However, levels of CS846 and COMP did not differ significantly between the placebo and proteoglycan groups at weeks 12 and 16 (Fig. 4D and E).

Assessment of JKOM and VAS scores. The test supplement was evaluated based on changes in the subscale scores of JKOM (33) and VAS. However, there were no significant differences between the placebo and proteoglycan groups during the intervention at weeks $4,8,12$ or 16 in the subscale scores of JKOM and VAS subscales, among the subjects of the initial analysis (Table I), the subjects with a total score of JKOM $\geq 41$ (Table II) and the subjects with pain at rest (Table III) (data not shown).

Together these observations suggest that oral administration of test supplement containing salmon nasal cartilage proteoglycan may have a protective effect on cartilage metabolism in subjects with severe and constant knee pain, by improving the $\mathrm{C} 1,2 \mathrm{C} / \mathrm{PIICP}$ ratio (lowering type II collagen degradation and increasing type II collagen synthesis).

Assessment of safety and tolerability. Out of all 60 enrolled subjects, $6(26.7 \%)$ in the placebo group and $11(36.7 \%)$ in the proteoglycan group experienced one or more adverse events during the intervention period. The total number of adverse events reported was 11 and 21 in the placebo and proteoglycan groups, respectively, and there was no significant difference in the frequency of adverse events occurring between the two groups $(\mathrm{P}=0.580)$. Major adverse events reported from the subjects of the placebo and proteoglycan groups were symptoms of a common cold (sore throat, cough and/or bronchitis), headache, back pain and neck/shoulder pain. All adverse events were of mild or moderate intensity and judged by the medical investigator to be unrelated to the intervention. 
A

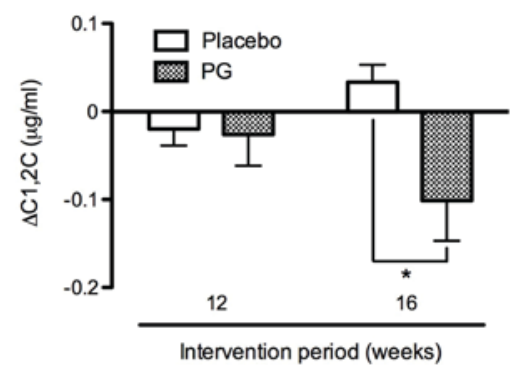

C

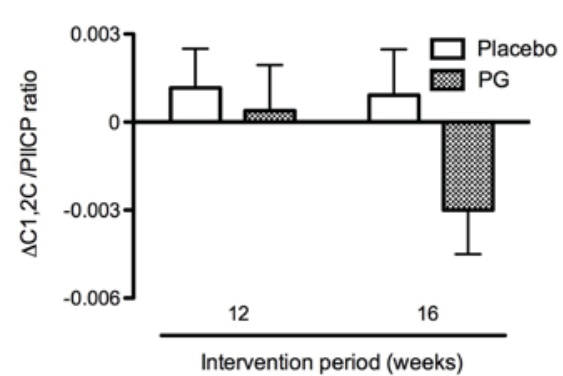

B
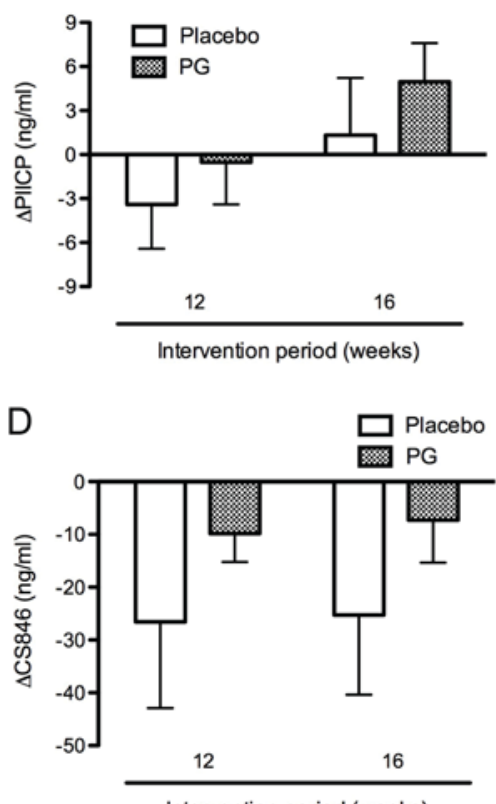

$\mathrm{E}$

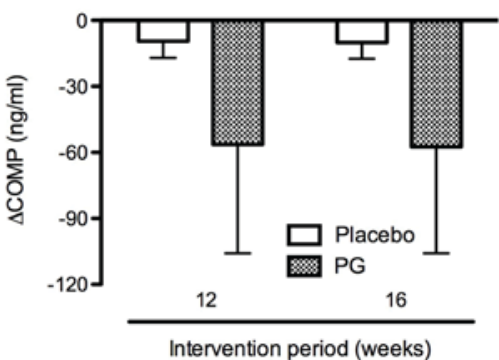

Figure 4. Cartilage metabolism in subjects with constant knee pain. Markers of cartilage metabolism were measured using sera collected from the subjects in the placebo $(n=12)$ and proteoglycan $(n=18)$ groups at baseline and at weeks 12 and 16 of the intervention. The marker levels were expressed as changes from the baseline. (A) $\Delta \mathrm{C} 1,2 \mathrm{C}$, (B) $\triangle \mathrm{PIICP},(\mathrm{C}) \Delta \mathrm{C} 1,2 \mathrm{C} / \mathrm{PIICP}$ ratio, (D) $\Delta \mathrm{CS} 486$ and (E) $\Delta \mathrm{COMP}$. ${ }^{*}<0.05$ vs. placebo. PIICP, C-terminal type II procollagen propeptide; COMP, cartilage oligometric matrix protein; $\mathrm{C} 1,2 \mathrm{C}$, marker of type II collagen degradation.

Furthermore, changes in physical measurement parameters (body weight and body mass index), physiological examinations (systolic and diastolic blood pressures, and pulse rate) and laboratory tests (urinalysis, hematology and blood chemistry) were minimal and within the reference values during the intervention in both groups.

\section{Discussion}

The biomarkers for cartilage metabolism, particularly type II collagen metabolism, are used to screen for the risk of progressive destruction of joint cartilage and also to monitor the effects of structure-modifying agents or dietary supplements on joint diseases, such as osteoarthritis $(8,9)$. For example, the actions of chondroprotective agents, (such as glucosamine and chondroitin sulfate), have been evaluated using type II collagen degradation biomarkers including CTX-II and C2C or $\mathrm{C} 1,2 \mathrm{C})(21,41-43)$. Type II collagen synthesis biomarkers, such as PIICP, have also been used in combination with type II collagen degradation biomarkers, including CTX-II and C2C or $\mathrm{C} 1,2 \mathrm{C}$, to monitor the disease state and progression of osteoarthritis. The combination of type II collagen degradation and synthesis biomarkers has been demonstrated to be more effective at predicting the progression of osteoarthritis or monitoring the action of chondroprotective agents on cartilage metabolism in osteoarthritis $(39,40,44)$.

The present study aimed to evaluate the action of salmon nasal cartilage proteoglycan on human joint health. A randomized double-blind placebo-controlled clinical trial was conducted to evaluate the effect of oral administration of proteoglycan $(10 \mathrm{mg} /$ day $)$ on cartilage metabolism in subjects with knee joint discomfort by measuring the serum levels of type II collagen degradation $(\mathrm{C} 1,2 \mathrm{C})$ and synthesis (PIICP) markers, as well as aggrecan (CS846) and COMP.

The $\mathrm{C} 1,2 \mathrm{C}$ antibody used to measure $\mathrm{C} 1,2 \mathrm{C}$ can recognize the cleaved fragment (neoepitope) of type I collagen as well as that of type II collagen in the cartilage, on the basis of sequence homology $(8,9)$. In this regard, the $\mathrm{C} 1,2 \mathrm{C}$ antibody has been used to detect the increased degradation of type I collagen in atheromatous plaques (45) and the modulation of type I collagen degradation in acute respiratory distress syndrome (46). However, in the present study, subjects 
with hypertension (atherosclerosis) and lung disorders were excluded. Thus, in the present study, serum levels of $\mathrm{C} 1,2 \mathrm{C}$ should reflect alterations in type II collagen metabolism in the cartilage.

The mechanism by which salmon nasal cartilage proteoglycan exerts protective action on cartilage metabolism remains unknown. Previous studies have reported that salmon nasal proteoglycan induces the proliferation of chondrocytes and the production of proteoglycans by chondrocytes in vitro $(29,30)$. The administration of salmon proteoglycan attenuated not only collagen-induced arthritis in mice, but also mouse experimental colitis in vivo by suppressing the expression of inflammatory cytokines, including interleukin (IL)-1 and IL-6 $(30,47)$. It has been determined that salmon nasal proteoglycan can be absorbed in the jejunum of the small intestine by clathrin-mediated endocytosis (48). Furthermore, type II collagen is degraded by collagenases, including MMP-1, MMP-8 and MMP-13, and the expression of these enzymes is upregulated by inflammatory cytokines produced in the articular cartilage $(49,50)$. Based on these findings, it is reasonable to speculate that orally administered salmon nasal cartilage proteoglycan is absorbed in the small intestine. Proteoglycan or its metabolites may exert anti-inflammatory actions via the suppression of inflammatory cytokine production, as well as a chondroprotective effect at the joint tissue via the proliferation of chondrocytes and the production of proteoglycans. This may inhibit the expression of MMPs and the degradation of type II collagen in the articular cartilage, as demonstrated by a decrease in C1,2C levels. However, the detailed mechanism for the chondroprotective action of salmon nasal cartilage proteoglycan in humans remains to be elucidated.

In the initial analysis of the results (Fig. 2), a statistically significant effect of salmon nasal cartilage proteoglycan on cartilage metabolism could not be detected. Thus, in the second and third analyses, only subjects with increased pain and physical dysfunction (total JKOM score $\geq 41$ ), and pain at rest were analyzed, and consequently salmon nasal cartilage proteoglycan was proved to be significantly effective at improving the cartilage metabolism. These observations suggested that additional inclusion criteria (such as subscales of JKOM and VAS), should be introduced when screening subjects in randomized double-blind placebo-controlled clinical trials to identify the potential action of dietary supplements on the cartilage metabolism in individuals with knee joint discomfort but without a diagnosis of osteoarthritis. In this context, it is interesting to note that a previous study by the current authors demonstrated that administration of salmon nasal cartilage proteoglycan (10 mg/day for 12 weeks) relieved the symptoms of osteoarthritis, including the subscale scores of JKOM and VAS, in subjects with knee osteoarthritis (29). By contrast, in the present study, the administration of salmon nasal proteoglycan (10 mg/day for 16 weeks) did not improve the symptoms of knee discomfort (evaluated by the subscale scores of JKOM and VAS) in subjects without the diagnosis of knee osteoarthritis. Notably, the total JKOM score (53.7 \pm 3.7$)$, VAS score of pain at rest $(33.7 \pm 5.7 \mathrm{~mm})$ and Kellgren and Lawrence grade (mainly 1 and 2) were higher in the subjects $(n=12)$ in the previous study (29), compared with subjects $(n=55)$ in the present study (total JKOM score, 46.4 \pm 1.2 ; VAS score of pain at rest, $20.4 \pm 3.5 \mathrm{~mm}$; Kellgren and Lawrence grade, mainly 0 and 1). Thus, salmon nasal cartilage proteoglycan is expected to more potently exert chondroprotective action and improve the knee joint discomfort in subjects experiencing greater levels of pain and dysfunction.

A previous open-label study demonstrated that the administration of salmon nasal cartilage proteoglycan relatively reduces the degradation and increases the synthesis of type II collagen, thereby improving the symptoms of osteoarthritis in individuals with knee osteoarthritis (30). However, the effect of the administration of salmon nasal proteoglycan on cartilage metabolism has not yet been confirmed in a blind study. Thus, to the best of our knowledge, this is the first randomized double-blind placebo-controlled clinical trial to demonstrate the effect of oral administration of salmon nasal proteoglycan on cartilage metabolism. The efficacy and safety of proteoglycan observed in the present study suggest that a dietary supplement containing salmon nasal proteoglycan is safe to administer and may exert a chondroprotective action in individuals with knee joint discomfort by improving type II collagen metabolism in cartilage. Thus, salmon nasal proteoglycan-containing supplement is a potential candidate for maintaining or improving joint health.

\section{Acknowledgements}

The authors would like to thank Mr. Takashi Nakagawa, Ms. Kaori Yoshimura and Professor Tetsuro Yamamoto (Total Technological Consultant Co., Ltd., Tokyo, Japan) for their helpful discussion and statistical expertise in the preparation of this manuscript.

The present study was funded by Ichimaru Pharcos Co., Ltd., which produces salmon nasal proteoglycan; however, the company had no input on the design and conduct of the study, subject recruitment, collection, management or analysis of the data. Dr Tatsuji Takahashi, Dr Yuka Tsuda Tanaka and Dr Makoto Tsuboi participated in the analyses of biomarkers as members of our research group and are employees of Ichimaru Pharcos Co., Ltd. Dr Akihito Tomonaga and Dr Kumie Ito performed the clinical study, and Professor Isao Nagaoka had control over the design of the study and preparation of the manuscript.

\section{References}

1. Ravenda V, Manette C, Lemmens R, Mariani AM, Struvay N and Reginster JY: Prevalence and impact of osteoarthritis and osteoporosis on health-related quality of life among active subjects. Aging Clin Exp Res 19: 55-60, 2007.

2. Jinks C, Jordan K and Croft P: Osteoarthritis as a public health problem: The impact of developing knee pain on physical function in adults living in the community: (KNEST 3). Rheumatology (Oxford) 46: 877-881, 2007.

3. Yoshimura N, Muraki S, Oka H, Mabuchi A, En-Yo Y, Yoshida M, Saika A, Yoshida H, Suzuki T, Yamamoto S, et al: Prevalence of knee osteoarthritis, lumbar spondylosis, and osteoporosis in Japanese men and women: The research on osteoarthritis/osteoporosis against disability study. J Bone Miner Metab 27: 620-628, 2009.

4. Qi C and Changlin H: Effects of moving training on histology and biomarkers levels of articular cartilage. J Surg Res 135: 352-363, 2006.

5. Garnero P, Rousseau JC and Delmas PD: Molecular basis and clinical use of biochemical markers of bone, cartilage, and synovium in joint diseases. Arthritis Rheum 43: 953-968, 2000. 
6. Garnero P, Piperno M, Gineyts E, Christgau S, Delmas PD and Vignon E: Cross sectional evaluation of biochemical markers of bone, cartilage and synovial tissue metabolism in patients with knee osteoarthritis: Relations with disease activity and joint damage. Ann Rheum Dis 60: 619-626, 2001.

7. Poole AR: Biochemical/immunochemical biomarkers of osteoarthritis: Utility for prediction of incident or progressive osteoarthritis. Rheum Dis Clin North Am 29: 803-818, 2003

8. Elsaid KA and Chichester CO: Review: Collagen markers in early arthritic diseases. Clin Chim Acta 365: 68-77, 2006.

9. Rousseau JC and Delmas PD: Biological markers in osteoarthritis. Nat Clin Pract Rheumatol 3: 346-356, 2007.

10. Garnero P and Delmas PD: Biomarkers in osteoarthritis. Curr Opin Rheumatol 15: 641-646, 2003.

11. Christgau S, Garnero P, Fledelius C, Moniz C, Ensig M, Gineyts E, Rosenquist C and Qvist P: Collagen type II C-telopeptide fragments as an index of cartilage degradation. Bone 29: 209-215, 2001.

12. Poole AR, Ionescu M, Fitzcharles MA and Billinghurst RC: The assessment of cartilage degradation in vivo: Development of an immunoassay for the measurement in body fluids of type II collagen cleaved by collagenases. J Immunol Methods 294 $145-153,2004$

13. Billinghurst RC, Dahlberg L, Ionescu M, Reiner A, Bourne R, Rorabeck C, Mitchell P, Hambor J, Diekmann O, Tschesche H, et al: Enhanced cleavage of type II collagen by collagenases in osteoarthritic articular cartilage. J Clin Invest 99: $1534-1545,1997$.

14. Shinmei M, Ito K, Matsuyama S, Yoshihara Y and Matsuzawa K Joint fluid carboxy-terminal type II procollagen peptide as a marker of cartilage collagen biosynthesis. Osteoarthritis Cartilage 1: 121-128, 1993.

15. Rizkalla G, Reiner A, Bogoch E and Poole AR: Studies of the articular cartilage proteoglycan aggrecan in health and osteoarthritis. Evidence for molecular heterogeneity and extensive molecular changes in disease. J Clin Invest 90: 2268-2277, 1992.

16. Lohmander LS, Ionescu M, Jugessur H and Poole AR: Changes in joint cartilage aggrecan after knee injury and in osteoarthritis Arthritis Rheum 42: 534-544, 1999.

17. Vilím V, Vytásek R, Olejárová M, Machácek S, Gatterová J, Procházka B, Kraus VB and Pavelka K: Serum cartilage oligomeric matrix protein reflects the presence of clinically diagnosed synovitis in patients with knee osteoarthritis. Osteoarthritis Cartilage 9: 612-618, 2001

18. Hunter DJ, Li J, LaValley M, Bauer DC, Nevitt M, DeGroot J, Poole R, Eyre D, Guermazi A, Gale D and Felson DT: Cartilage markers and their association with cartilage loss on magnetic resonance imaging in knee osteoarthritis: The Boston Osteoarthritis Knee Study. Arthritis Res Ther 9: R108, 2007.

19. Jansen NW, Roosendaal G, Lundin B, Heijnen L, Mauser-Bunschoten E, Bijlsma JW, Theobald M and Lafeber FP: The combination of the biomarkers urinary $\mathrm{C}$-terminal telopeptide of type II collagen, serum cartilage oligomeric matrix protein and serum chondroitin sulfate 846 reflects cartilage damage in hemophilic arthropathy. Arthritis Rheum 60: 290-298, 2009.

20. Clayton JJ: Nutraceuticals in the management of osteoarthritis Orthopedics 30: 624-631, 2007.

21. Nagaoka I: Recent aspects of the chondroprotective and anti-Inflammatory actions of glucosamine, a functional food Juntendo Med J 60: 580-587, 2014

22. Fenton JI, Chlebek-Brown KA, Peters TL, Caron JP and Orth MW: Glucosamine $\mathrm{HCl}$ reduces equine articular cartilage degradation in explant culture. Osteoarthritis Cartilage 8: $258-265,2000$

23. Gouze JN, Bordji K, Gulberti S, Terlain B, Netter P, Magdalou J, Fournel-Gigleux S and Ouzzine M: Interleukin-1beta downregulates the expression of glucuronosyltransferase I, a key enzyme priming glycosaminoglycan biosynthesis: Influence of glucosamine on interleukin-lbeta-mediated effects in rat chondrocytes Arthritis Rheum 44: 351-360, 2001.

24. Nakamura H, Shibakawa A, Tanaka M, Kato T and Nishioka K Effects of glucosamine hydrochloride on the production of prostaglandin E2, nitric oxide and metalloproteases by chondrocytes and synoviocytes in osteoarthritis. Clin Exp Rheumatol 22: 293-299, 2004

25. Derfoul A, Miyoshi AD, Freeman DE and Tuan RS: Glucosamine promotes chondrogenic phenotype in both chondrocytes and mesenchymal stem cells and inhibits MMP-13 expression and matrix degradation. Osteoarthritis Cartilage 15: 646-655, 2007.
26. McAlindon TE, Lavalley MP, Gulin JP and Felson DT: Glucosamine and chondroitin for treatment of osteoarthritis: A systematic quality assessment and meta-analysis. JAMA 283: $1469-1475,2000$

27. Reginster JY, Deroisy R, Rovati LC, Lee RL, Lejeune E, Bruyere O, Giacovelli G, Henrotin Y, Dacre JE and Gossett C: Long-term effects of glucosamine sulphate on osteoarthritis progression: A randomized, placebo-controlled clinical trial. Lancet 357: 251-256, 2001.

28. Pavelká K, Gatterová J, Olejarová M, Machacek S, Giacovelli G and Rovati LC: Glucosamine sulfate use and delay of progression of knee osteoarthritis: A 3-year, randomized, placebo-controlled, double-blind study. Arch Intern Med 162: 2113-2123, 2002.

29. Takahashi T, Masutani T, Tomonaga A, Watanabe K, Yamamoto T, Ito K, Tsuboi M, Yamaguchi $\mathrm{H}$ and Nagaoka I: Influence on improvement of osteoarthritis by oral intake of proteoglycan extracted from salmon nasal cartilage. In: Proceedings of the International Conference and Exhibition on Nutraceuticals and Functional Foods. Sapporo, Abstract P112, 2011.

30. Ohshika S, Ishibashi Y, Kon A, Kusumi T, Kijima H and Toh S: Potential of exogenous cartilage proteoglycan as a new material for cartilage regeneration. Int Orthop 36: 869-877, 2012.

31. Yoshimura S, Asano $K$ and Nakane A: Attenuation of collagen-induced arthritis in mice by salmon proteoglycan. Biomed Res Int 2014: 406453, 2014

32. Kellgren JH and Lawrence JS: Radiological assessment of osteo-arthritis. Ann Rheum Dis 16: 494-502, 1957.

33. Akai M, Iwaya T, Kurosawa H, Doi T, Nasu T, Hayashi K and Fujino K: Development of new disease-specific QOL measure for patients with knee osteoarthritis: Japanese knee osteoarthritis measure (JKOM). J Phys Med 16: 55-62, 2005 (In Japanese).

34. Okuda M, Omokawa S, Okahashi K, Akahane M and Tanaka Y: Validity and reliability of the Japanese Orthopaedic Association score for osteoarthritic knees. J Orthop Sci 17: 750-756, 2012.

35. Majima M, Takagaki K, Sudo S, Yoshihara S, Kudo Y and Yamagishi S: Effect of proteoglycan on experimental colitis. Int Cong Ser 1223: 221-224, 2001.

36. Takahashi T, Matsubara J, Wakamatsu K, Tsuda Tanaka Y, Masutani T, Yonezuka M, Ito K, Tsuji-Takayama K and Tsuboi M Ingestion of salmon nasal cartilage-derived proteoglycan improves skin condition: A randomized, double-blind, controlled study. Immun Endoc Metab Agents Med Chem 15: 160-167, 2015.

37. Takahashi T, Maeda M, Matsubara J, Fujita Y, Masutani T, Yonezuka M, Ito K, Tsuji-Takayama K and Tsuboi M: Safety evaluation of industrially extracted, highly purified proteoglycan from salmon nasal cartilatge. Pharmacometrics 89: 15-22, 2015.

38. Akai M, Doi T, Fujino K, Iwaya T, Kurosawa H and Nasu T: An outcome measure for Japanese people with knee osteoarthritis. J Rheumatol 32: 1524-1532, 2005.

39. Cahue S, Sharma L, Dunlop D, Ionescu M, Song J, Lobanok T, King L and Poole AR: The ratio of type II collagen breakdown to synthesis and its relationship with the progression of knee osteoarthritis. Osteoarthr Cartil 15: 819-823, 2007.

40. Sharif M, Kirwan J, Charni N, Sandell LJ, Whittles C and Garnero P: A 5-yr longitudinal study of type IIA collagen synthesis and total type II collagen degradation in patients with knee osteoarthritis-association with disease progression. Rheumatology (Oxford) 46: 938-943, 2007.

41. Christgau S, Henrotin Y, Tankó LB, Rovati LC, Collette J, Bruyere O, Deroisy R and Reginster JY: Osteoarthritic patients with high cartilage turnover show increased responsiveness to the cartilage protecting effects of glucosamine sulfate. Clin Exp Rheumatol 22: 36-42, 2004.

42. Cibere J, Thorne A, Kopec JA, Singer J, Canvin J, Robinson DB, Pope J, Hong P, Grant E, Lobanok T, et al: Glucosamine sulfate and cartilage type II collagen degradation in patients with knee osteoarthritis. J Rheumatol 32: 896-902, 2005.

43. Mazières B, Hucher M, Zaïm M and Garnero P: Effect of chondroitin sulfate in symptomatic knee osteoarthritis: A multicentre, randomised, double-blind, placebo-controlled study. Ann Rheum Dis 66: 639-645, 2007.

44. Conrozier T, Poole AR, Ferrand F, Mathieu P, Vincent F, Piperno M, Verret C, Ionescu M and Vignon E: Serum concentrations of type II collagen biomarkers (C2C, C1, 2C and CPII) suggest different pathophysiologies in patients with hip osteoarthritis. Clin Exp Rheumatol 26: 430-435, 2008.

45. Sukhova GK, Schönbeck U, Rabkin E, Schoen FJ, Poole AR, Billinghurst RC and Libby P: Evidence for increased collagenolysis by interstitial collagenases-1 and -3 in vulnerable human atheromatous plaques. Circulation 99: 2503-2509, 1999. 
46. Armstrong L, Tickett DR, Mansell JP, Ionescu M, Hoyle E, Billinghurst RC, Poole AR and Millar AB: Changes in collagen turnover in early acute respiratory distress syndrome. Am J Respir Crit Care Med 160: 1910-1915, 1999.

47. Mitsui T, Sashinami H, Sato F, Kijima H, Ishiguro Y, Fukuda S, Yoshihara S, Hakamada K and Nakane A: Salmon cartilage proteoglycan suppresses mouse experimental colitis through induction of Foxp3 ${ }^{+}$regulatory $\mathrm{T}$ cells. Biochem Biophys Res Commun 402: 209-215, 2010.

48. Tsuchiya Y, Tomita M, Tsuboi M, Takahashi Y, Yonezuka M, Kikuchi S, Nagasawa S, Kumazawa A and Kubota J: Absorption of proteoglycan via clathrin-mediated endocytosis in the small intestine of rats. Biosci Biotechnol Biochem 77: 654-656, 2013.
49. Billinghurst RC, $\mathrm{Wu} \mathrm{W}$, Ionescu M, Reiner A, Dahlberg L, Chen J, van Wart $\mathrm{H}$ and Poole AR: Comparison of the degradation of type II collagen and proteoglycan in nasal and articular cartilages induced by interleukin-1 and the selective inhibition of type II collagen cleavage by collagenase. Arthritis Rheum 43: 664-672, 2000

50. Rousset F, Hazane-Puch F, Pinosa C, Nguyen MV, Grange L, Soldini A, Rubens-Duval B, Dupuy C, Morel F and Lardy B: IL-1beta mediates MMP secretion and IL-1beta neosynthesis via upregulation of p22(phox) and NOX4 activity in human articular chondrocytes. Osteoarthritis Cartilage 23: 1972-1980, 2015. 